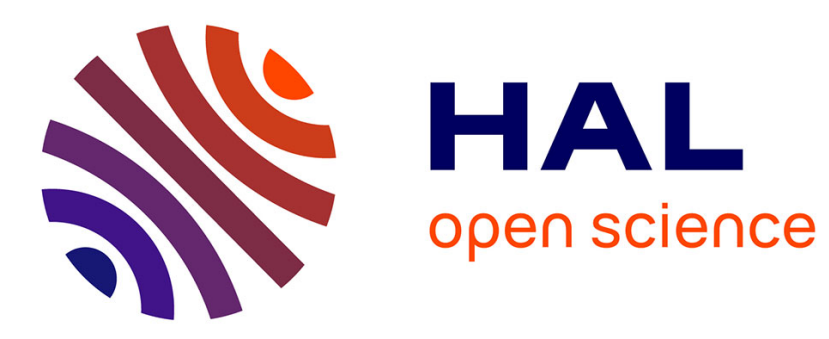

\title{
Fixed-Time Output Stabilization of a Chain of Integrators
}

Francisco Lopez-Ramirez, Denis Efimov, Andrey Polyakov, Wilfrid

Perruquetti

\section{- To cite this version:}

Francisco Lopez-Ramirez, Denis Efimov, Andrey Polyakov, Wilfrid Perruquetti. Fixed-Time Output Stabilization of a Chain of Integrators. Proc. 55th IEEE Conference on Decision and Control (CDC), Dec 2016, Las Vegas, United States. hal-01371278

\section{HAL Id: hal-01371278 \\ https://hal.inria.fr/hal-01371278}

Submitted on 25 Sep 2016

HAL is a multi-disciplinary open access archive for the deposit and dissemination of scientific research documents, whether they are published or not. The documents may come from teaching and research institutions in France or abroad, or from public or private research centers.
L'archive ouverte pluridisciplinaire HAL, est destinée au dépôt et à la diffusion de documents scientifiques de niveau recherche, publiés ou non, émanant des établissements d'enseignement et de recherche français ou étrangers, des laboratoires publics ou privés. 


\title{
Fixed-Time Output Stabilization of a Chain of Integrators
}

\author{
F. Lopez-Ramirez, D. Efimov, A. Polyakov, W. Perruquetti
}

\begin{abstract}
A solution to the problem of global fixed-time output stabilization of a chain of integrators is proposed. A nonlinear state feedback and a dynamic observer are designed in order to guarrantee both fixed-time estimation and fixedtime control. Robustness with respect to exogenous disturbances and measurement noises is established. The performance of the obtained control and estimation algorithms are illustrated by numeric experiments.
\end{abstract}

\section{INTRODUCTION}

Feedback stabilization of linear and nonlinear systems is one of the central problems in control systems theory. There are many methods to design a control [1], [2], [3], which differ by the requirements imposed on the plant model and on the guaranteed performance of the closed-loop system. Among performance criteria the robustness with respect to external disturbances or measurement noises and the rate of convergence are the most popular. To assess robustness of nonlinear systems the input-to-state stability theory is frequently used [4], [5]. The rate of convergence to the goal state or set can be asymptotic (e.g. exponential) or nonasymptotic, i.e. finite-time or fixed-time [6], [7], [8]. In the latter case the system converges uniformly from any initial conditions of the domain of attraction in a finite time (see more rigorous definitions below).

The present work studies the problem of output feedback design providing a fixed-time rate of convergence for a chain of integrators [9]. The chain of integrators is a very versatile and well studied system since all linear controllable systems and many nonlinear ones can be transformed, through a linear coordinate transformation in the first case and through feedback linearization in the second, into this particular form [10], [11]. Fixed-time stability, i.e. globally bounded convergence rate, is a relatively recent topic [12], [13], [14] and in [15] a fixed-time feedback regulator for a chain of integrators is presented based in Lyapunov analysis. Here we present a full control scheme, endowed with a state and

All authors are with Inria, Non-A team, Parc Scientifique de la Haute Borne, 40 avenue Halley, 59650 Villeneuve d'Ascq, France and CRIStAL (UMR-CNRS 9189), École Centrale de Lille, Cité Scientifique, 59651 Villeneuve-d'Ascq, France.

D. Efimov and A. Polyakov are with Department of Control Systems and Informatics, University ITMO, 49 av. Kronverkskiy, 197101 Saint Petersburg, Russia.

This work was partially supported by ANR Project Finite4SOS (ANR 15CE23-0007), Project GEC-Beihang 2015 (Laboratoire international associé), the Government of Russian Federation (Grant 074-U01) and the Ministry of Education and Science of Russian Federation (Project 14.Z50.31.0031). output feedback control as well as with a state observer, all of whom exhibit the fixed-time stability property. Instead of using a Lyapunov approach, the proposed solution is based on homogeneity theory [3], [16]; and contrarily to [9], the presented control has an explicit form. Furthermore we present a robustness analysis of the control scheme with respect to bounded external disturbances and measurement noises.

The outline of this paper is as follows. Notation and preliminary results are introduced in sections II and III. The precise problem statement is given in Section IV. The proposed algorithms are presented in Section $\mathrm{V}$ and a numerical example that illustrates the main features of the algorithms is given in Section VI. Concluding remarks and the discussion appear in Section VII.

\section{NOTATION}

Throughout the paper the following notation is used:

- $\mathbb{R}_{+}=\{x \in \mathbb{R}: x \geq 0\}$, where $\mathbb{R}$ is the set of real numbers.

- $|\cdot|$ denotes the absolute value in $\mathbb{R},\|$.$\| denotes the$ Euclidean norm on $\mathbb{R}^{n},\|x\|_{\mathcal{A}}=\inf _{\xi \in \mathcal{A}}\|x-\xi\|$ is the distance from a point $x \in \mathbb{R}^{n}$ to a set $\mathcal{A} \subset \mathbb{R}^{n} ;\|A\|_{2}$ is the induced matrix norm for $A \in \mathbb{R}^{n \times n}$.

- For a (Lebesgue) measurable function $d: \mathbb{R}_{+} \rightarrow$

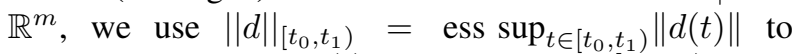
define the norm of $d(t)$ in the interval $\left[t_{0}, t_{1}\right)$, then $\|d\|_{\infty}=\|d\|_{[0,+\infty)}$ and the set of $d(t)$ with the property $\|d\|_{\infty}<+\infty$ is the set of essentially bounded measurable functions, we further denote this set as $\mathcal{L}_{\infty}$ $; \mathcal{L}_{D}=\left\{d \in \mathcal{L}_{\infty}:\|d\|_{\infty} \leq D\right\}$ for any $D>0$.

- A continuous function $\alpha: \mathbb{R}_{+} \rightarrow \mathbb{R}_{+}$belongs to the class $\mathcal{K}$ if $\alpha(0)=0$ and the function is strictly increasing. The function $\alpha: \mathbb{R}_{+} \rightarrow \mathbb{R}_{+}$belongs to the class $\mathcal{K}_{\infty}$ if $\alpha \in \mathcal{K}$ and it is increasing to infinity. A continuous function $\beta: \mathbb{R}_{+} \times \mathbb{R}_{+} \rightarrow \mathbb{R}_{+}$belongs to the class $\mathcal{K} \mathcal{L}$ if $\beta(\cdot, t) \in \mathcal{K}_{\infty}$ for each fixed $t \in \mathbb{R}_{+}$and $\lim _{t \rightarrow+\infty} \beta(s, t)=0$ for each fixed $s \in \mathbb{R}_{+}$.

- The notation $D V(x) f(x)$ stands for the directional derivative of a continuously differentiable function $V$ with respect to the vector field $f$ evaluated at point $x$.

- A series of integers $1,2, \ldots, n$ is denoted by $\overline{1, n}$. 


\section{PReliminaries}

Consider the following nonlinear system

$$
\dot{x}(t)=f(x(t), d(t)), t \geq 0,
$$

where $x(t) \in \mathbb{R}^{n}$ is the state, $d(t) \in \mathbb{R}^{m}$ is the input, $d \in \mathcal{L}_{\infty} ; f: \mathbb{R}^{n+m} \rightarrow \mathbb{R}^{n}$ ensures forward existence of the system solutions at least locally, $f(0,0)=0$. For an initial condition $x_{0} \in \mathbb{R}^{n}$ and input $d \in \mathcal{L}_{\infty}$ define the corresponding solution by $X\left(t, x_{0}, d\right)$ for any $t \geq 0$ for which the solution exists.

Following [7], [10], [8], let $\Omega$ be an open neighborhood of the origin in $\mathbb{R}^{n}$ and $D>0$.

Definition 1. At the steady state $x=0$ the system (1) for $d \in \mathcal{L}_{D}$ is said to be

(a) uniformly Lyapunov stable if for any $x_{0} \in \Omega$ and $d \in \mathcal{L}_{D}$ the solution $X\left(t, x_{0}, d\right)$ is defined for all $t \geq 0$, and for any $\epsilon>0$ there is $\delta>0$ such that for any $x_{0} \in \Omega$, if $\left\|x_{0}\right\| \leq \delta$ then $\left\|X\left(t, x_{0}, d\right)\right\| \leq \epsilon$ for all $t \geq 0$;

(b) uniformly asymptotically stable if it is uniformly Lyapunov stable and for any $\kappa>0$ and $\epsilon>0$ there exists $T(\kappa, \epsilon) \geq 0$ such that for any $x_{0} \in \Omega$ and $d \in \mathcal{L}_{D}$, if $\left\|x_{0}\right\| \leq \kappa$ then $\left\|X\left(t, x_{0}, d\right)\right\| \leq \epsilon$ for all $t \geq T(\kappa, \epsilon)$;

(c) uniformly finite-time stable if it is uniformly Lyapunov stable and uniformly finite-time converging from $\Omega$, i.e. for any $x_{0} \in \Omega$ and all $d \in \mathcal{L}_{D}$ there exists $0 \leq T<+\infty$ such that $X\left(t, x_{0}, d\right)=0$ for all $t \geq T$. The function $T_{0}\left(x_{0}\right)=\inf \left\{T \geq 0: X\left(t, x_{0}, d\right)=0 \forall t \geq T, \forall d \in \mathcal{L}_{D}\right\}$ is called the uniform settling time of the system (1);

(d) uniformly fixed-time stable if it is uniformly finitetime stable and $\sup _{x_{0} \in \Omega} T_{0}\left(x_{0}\right)<+\infty$.

The set $\Omega$ is called the domain of stability/attraction.

If $\Omega=\mathbb{R}^{n}$, then the corresponding properties are called global uniform Lyapunov/asymptotic/finite-time/fixedtime stability of (1) for $d \in \mathcal{L}_{D}$ at $x=0$.

Stability notions can be similarly defined with respect to a set, by replacing the distance to the origin in Definition 1 with the distance to an invariant set. For example, the global uniform finite-time stability with respect to a set $\mathcal{A} \subset \mathbb{R}^{n}$ is equivalent to the following two properties:

(i) uniform Lyapunov stability: for any $x_{0} \in \mathbb{R}^{n}$ and $d \in \mathcal{L}_{D}$ the solution $X\left(t, x_{0}, d\right)$ is defined for all $t \geq 0$, and for any $\epsilon>0$ there is $\delta>0$ such that if $\left\|x_{0}\right\|_{\mathcal{A}} \leq \delta$ then $\left\|X\left(t, x_{0}, d\right)\right\|_{\mathcal{A}} \leq \epsilon$ for all $t \geq 0$;

(ii) uniform finite-time convergence: for any $x_{0} \in \mathbb{R}^{n}$ and all $d \in \mathcal{L}_{D}$ there exists $0 \leq T<+\infty$ such that $\left\|X\left(t, x_{0}, d\right)\right\|_{\mathcal{A}}=0$ for all $t \geq T$.

\section{A. Input-to-state stability}

More details about this theory can be found in [5].

Definition 2. The system (1) is called input-to-state practically stable (ISpS), if for any input $d \in \mathcal{L}_{\infty}$ and any $x_{0} \in \mathbb{R}^{n}$ there are some functions $\beta \in \mathcal{K} \mathcal{L}, \gamma \in \mathcal{K}$ and $c \geq 0$ such that

$$
\left\|X\left(t, x_{0}, d\right)\right\| \leq \beta\left(\left\|x_{0}\right\|, t\right)+\gamma\left(\|d\|_{[0, t)}\right)+c \quad \forall t \geq 0 .
$$

The function $\gamma$ is called nonlinear asymptotic gain. The system is called input-to-state stable (ISS) if $c=0$.

Definition 3. A smooth function $V: \mathbb{R}^{n} \rightarrow \mathbb{R}_{+}$is called ISpS Lyapunov function for the system (1) if for all $x \in \mathbb{R}^{n}$, $d \in \mathbb{R}^{m}$ and some $r \geq 0, \alpha_{1}, \alpha_{2}, \alpha_{3} \in \mathcal{K}_{\infty}$ and $\theta \in \mathcal{K}$ :

$$
\begin{aligned}
\alpha_{1}(\|x\|) & \leq V(x) \leq \alpha_{2}(\|x\|), \\
D V(x) f(x, d) & \leq r+\theta(\|d\|)-\alpha_{3}(\|x\|) .
\end{aligned}
$$

Such a function $V$ is called ISS Lyapunov function if $r=0$.

Note that an ISS Lyapunov function can also satisfy the following equivalent condition for some $\chi \in \mathcal{K}_{\infty}$ :

$$
\|x\|>\chi(\|d\|) \Rightarrow D V(x) f(x, d) \leq-\alpha_{3}(\|x\|) .
$$

Theorem 1. The system (1) is ISS (ISpS) iff it admits an ISS (ISpS) Lyapunov function.

\section{B. Weighted homogeneity}

Following [17], [3], for strictly positive numbers $r_{i}, i=$ $\overline{1, n}$ called weights and $\lambda>0$, one can define:

- the vector of weights $\mathbf{r}=\left(r_{1}, \ldots, r_{n}\right)^{T}, r_{\max }=$ $\max _{1 \leq j \leq n} r_{j}$ and $r_{\min }=\min _{1 \leq j \leq n} r_{j}$;

- the dilation matrix function $\Lambda_{r}(\bar{\lambda})=\operatorname{diag}\left\{\lambda^{r_{i}}\right\}_{i=1}^{n}$, note that $\forall x \in \mathbb{R}^{n}$ and $\forall \lambda>0$ we have $\Lambda_{r}(\lambda) x=$ $\left(\lambda^{r_{1}} x_{1}, \ldots, \lambda^{r_{i}} x_{i}, \ldots, \lambda^{r_{n}} x_{n}\right)^{T}$;

- the r-homogeneous norm $\|x\|_{r}=\left(\sum_{i=1}^{n}\left|x_{i}\right|^{\frac{\rho}{r_{i}}}\right)^{\frac{1}{\rho}}$ for any $x \in \mathbb{R}^{n}$ and $\rho \geq r_{\max }$, then there exist $\underline{\sigma}, \bar{\sigma} \in \mathcal{K}_{\infty}$ such that

$$
\underline{\sigma}\left(\|x\|_{r}\right) \leq\|x\| \leq \bar{\sigma}\left(\|x\|_{r}\right) \quad \forall x \in \mathbb{R}^{n} .
$$

- the unit sphere and a ball in the homogeneous norm $S_{r}=\left\{x \in \mathbb{R}^{n}:\|x\|_{r}=1\right\}$ and $B_{r}(\rho)=\left\{x \in \mathbb{R}^{n}:\right.$ $\left.\|x\|_{r} \leq \rho\right\}$ for $\rho \geq 0$.

Definition 4. A function $g: \mathbb{R}^{n} \rightarrow \mathbb{R}$ is $\mathbf{r}$-homogeneous with degree $\eta \in \mathbb{R}$ if $\forall x \in \mathbb{R}^{n}$ and $\forall \lambda>0$ we have:

$$
\lambda^{-\eta} g\left(\Lambda_{r}(\lambda) x\right)=g(x) .
$$

A vector field $\phi: \mathbb{R}^{n} \rightarrow \mathbb{R}^{n}$ is $\mathbf{r}$-homogeneous with degree $\nu \in \mathbb{R}$, with $\nu \geq-r_{\text {min }}$ if $\forall x \in \mathbb{R}^{n}$ and $\forall \lambda>0$ we have:

$$
\lambda^{-\nu} \Lambda_{r}^{-1}(\lambda) \phi\left(\Lambda_{r}(\lambda) x\right)=\phi(x),
$$

which is equivalent to the $i$-th component of $\phi$ being a $\mathbf{r}$-homogeneous function of degree $r_{i}+\nu$.

The system (1) for $d=0$ is $\mathbf{r}$-homogeneous of degree $\nu$ if the vector field $f$ is $\mathbf{r}$-homogeneous of degree $\nu$ for $d=0$.

The property of $\mathbf{r}$-homogeneity can also be defined not for all $x \in \mathbb{R}^{n}$ but for a subset of the state space (or it can be approximately satisfied [18], [19]).

An important advantage of $\mathbf{r}$-homogeneous systems is that their rate of convergence can be evaluated qualitatively based on their degree of homogeneity.

Lemma 1. [3] If (1) for $d=0$ is $\mathbf{r}$-homogeneous of degree $\nu$ and asymptotically stable at the origin, then it is 
(i) globally finite-time stable at the origin if $\nu<0$;

(ii) globally exponentially stable at the origin if $\nu=0$;

(iii) globally fixed-time stable with respect to the unit ball $B_{r}(1)$ if $\nu>0$.

Define $\tilde{f}(x, d)=\left[\begin{array}{ll}f(x, d)^{T} & 0_{m}\end{array}\right]^{T} \in \mathbb{R}^{n+m}$, it is an extended auxiliary vector field for the system (1), where $0_{m}$ is the zero vector of dimension $m$.

Theorem 2. [20] Let the vector field $\tilde{f}$ be homogeneous with the weights $\mathbf{r}=\left[r_{1}, \ldots, r_{n}\right]>0, \tilde{\mathbf{r}}=\left[\tilde{r}_{1}, \ldots, \tilde{r}_{m}\right]>0$ with a degree $\nu \geq-r_{\min }$, i.e. $f\left(\Lambda_{r}(\lambda) x, \Lambda_{\tilde{r}}(\lambda) d\right)=$ $\lambda^{\nu} \Lambda_{r}(\lambda) f(x, d)$ for all $x \in \mathbb{R}^{n}, d \in \mathbb{R}^{m}$ and all $\lambda>0$. Assume that the system (1) is globally asymptotically stable for $d=0$, then the system (1) is ISS.

Therefore, for the homogeneous system (1), its ISS property follows from its asymptotic stability for $d=0$ (as for linear systems [5]).

\section{Problem Statement}

Consider a chain of integrators:

$$
\begin{aligned}
\dot{x}(t) & =A_{0} x(t)+b u(t)+d(t), t \geq 0, \\
y(t) & =C x(t)+v(t),
\end{aligned}
$$

where $x(t) \in \mathbb{R}^{n}$ is the state vector, $u(t) \in \mathbb{R}$ is the control input, $y(t) \in \mathbb{R}$ is the measured output; $d(t) \in \mathbb{R}^{n}$ and $v(t) \in$ $\mathbb{R}$ are the exogenous disturbance and the measurement noise, respectively, $(d, v) \in \mathcal{L}_{\infty}$; the matrices

$$
A_{0}=\left[\begin{array}{cccc}
0 & 1 & 0 \ldots 0 & 0 \\
0 & 0 & 1 \ldots 0 & 0 \\
\vdots & \vdots & \ddots & \vdots \\
0 & 0 & 0 \ldots 0 & 1 \\
0 & 0 & 0 \ldots 0 & 0
\end{array}\right], b=\left[\begin{array}{c}
0 \\
\vdots \\
0 \\
1
\end{array}\right], C=\left[\begin{array}{lll}
1 & 0 \ldots 0
\end{array}\right],
$$

are in the canonical form.

It is required to design a stabilizing dynamic output control $u$ that ensures the ISpS property of the system (2) for any $(d, v) \in \mathcal{L}_{\infty}$; and that for $d=v=0$ provides global fixedtime stability of the closed-loop system at the origin.

\section{Main Result}

The solution of the problem is divided in three steps. First, a state feedback is proposed ensuring the problem solution. Second, the equations of the observer are introduced. Third, a combined output feedback is presented and analyzed.

\section{A. State feedback}

For $x \in \mathbb{R}$ and $\alpha>0$ denote $\lceil x\rfloor^{\alpha}=|x|^{\alpha} \operatorname{sign}(x)$, then the control proposed in this work has the form:

$$
\begin{gathered}
u(x)=\sum_{i=1}^{n} a_{i}\left\lceil x_{i}\right\rfloor^{\alpha_{i}(\nu(\|x\|))}, \\
\alpha_{i}(\nu)=\frac{1+n \nu}{1+(i-1) \nu} \quad i=\overline{1, n},
\end{gathered}
$$

$$
\nu(s)= \begin{cases}\nu_{1} & \text { if } s \leq m, \\ \nu_{2} & \text { if } s \geq M \\ \frac{\nu_{2}-\nu_{1}}{M-m} s+\frac{M \nu_{1}-m \nu_{2}}{M-m} & \text { otherwise }\end{cases}
$$

where $a=\left[a_{1}, \ldots, a_{n}\right]^{T}$ is the vector of control coefficients forming a Hurwitz polynomial, $-\infty<\nu_{1}<0<\nu_{2}<+\infty$ and $0<m<M<+\infty$ are the tuning parameters to be defined later. Denote

$$
r_{i}(\nu)=1+(i-1) \nu \quad i=\overline{1, n},
$$

then it is straightforward to verify that for $d=0$ the system (2) is $\mathbf{r}\left(\nu_{1}\right)$-homogeneous of degree $\nu_{1}<0$ for $\|x\| \leq m$ and $\mathbf{r}\left(\nu_{2}\right)$-homogeneous of degree $\nu_{2}>0$ for $\|x\| \geq M$. Let us show that for a properly selected control parameters (2), (3) is globally fixed-time stable at the origin.

Lemma 2. Let $a \in \mathbb{R}^{n}$ form a Hurwitz polynomial, then for any $0<m<M<+\infty$ there exists $\epsilon \in\left(0, n^{-1}\right)$ such that if $\nu_{1} \in(-\epsilon, 0)$ and $\nu_{2} \in(0, \epsilon)$ then the system (2), (3) for $d=0$ is globally fixed-time stable at the origin.

Proof's sketch. Denote

$$
A=A_{0}+b a^{T}=\left[\begin{array}{cccccc}
0 & 1 & 0 & \ldots & 0 & 0 \\
0 & 0 & 1 & \ldots & 0 & 0 \\
\vdots & \vdots & \vdots & \ddots & 1 & \vdots \\
0 & 0 & 0 & \ldots & 0 & 1 \\
a_{1} & a_{3} & a_{3} & \ldots & a_{n-1} & a_{n}
\end{array}\right],
$$

then by the lemma conditions there are matrices $P \in \mathbb{R}^{n \times n}$ and $Q \in \mathbb{R}^{n \times n}$ such that

$$
P=P^{T}>0, Q=Q^{T}>0, A^{T} P+P A=-Q .
$$

Consider for (2), (3) a Lyapunov function $V(x)=x^{T} P x$, whose derivative admits the differential equation

$$
\dot{V}(x)=D V(x)\left[A_{0} x+b u(x)\right]=-x^{T} Q x+2 x^{T} P b \delta(x),
$$

where $\delta(x)=\sum_{i=1}^{n} a_{i}\left(\left\lceil x_{i}\right\rfloor^{\alpha_{i}(\nu(\|x\|))}-x_{i}\right)$. By construction, $\delta(x)=0$ iff $x=0$ or $\nu(\|x\|)=0$, i.e. for $\|x\|=0$ or $\|x\|=\mu=\frac{m \nu_{2}-M \nu_{1}}{\nu_{2}-\nu_{1}}$. Since $\delta: \mathbb{R}^{n} \rightarrow \mathbb{R}$ is a continuous function and $\nu^{\nu_{2}-\nu_{1}}: \mathbb{R}_{+} \rightarrow\left[\nu_{1}, \nu_{2}\right]$, it is possible to select the values of $\nu_{1}$ and $\nu_{2}$ sufficiently close to zero such that $\dot{V}(x)<0(|\delta(x)|$ stays small enough) on any compact containing the level $\|x\|=\mu$. Using the Lemma 1, we can prove that the system is $\mathbf{r}\left(\nu_{1}\right)$-homogeneous of degree $\nu_{1}<0$ and finite-time stable at the origin with respect to $B_{r\left(\nu_{1}\right)}\left(\rho_{1}\right)$ for $B_{r\left(\nu_{1}\right)}\left(\rho_{1}\right) \subset\left\{x \in \mathbb{R}^{n}:\|x\| \leq m\right\}$, and it is $\mathbf{r}\left(\nu_{2}\right)$-homogeneous of degree $\nu_{2}>0$ and globally fixed-time stable with respect to any ball $B_{r\left(\nu_{2}\right)}\left(\rho_{2}\right)$ such that $\left\{x \in \mathbb{R}^{n}:\|x\|=M\right\} \subset B_{r\left(\nu_{2}\right)}\left(\rho_{2}\right)$. Remark that $M$ and $n$ can be selected in a such a way that the time spent by the system's solutions in the set $\left\{x \in \mathbb{R}^{n}: m \leq\|x\| \leq M\right\}$ is finite. Gathering this arguments we conclude that (2), (3) is globally convergent and globally fixed-time stable at the origin. 
In order to analyze robust stability properties of the closed loop dynamics (2), (3) let us introduce

$$
f_{\nu}(x, \tilde{d})=A_{0} x+b \sum_{i=1}^{n} a_{i}\left[x_{i}+\tilde{d}_{1, i}\right\rfloor^{\alpha_{i}(\nu)}+\tilde{d}_{2},
$$

where $\tilde{d}=\left[\begin{array}{ll}\tilde{d}_{1}^{T} & \tilde{d}_{2}^{T}\end{array}\right]^{T} \in \mathbb{R}^{2 n}$ is the new disturbance input, $\tilde{d}_{1}$ represents measurements noises and $\tilde{d}_{2}=d$.

Corollary 1. Let all conditions of Lemma 2 be satisfied, then the system (2), (3) is ISpS for any $\tilde{d} \in \mathcal{L}_{\infty}$.

Proof's sketch. Consider the system (2), (3) for $\|x\| \geq$ $M$, then $\dot{x}=f_{\nu_{2}}(x, \tilde{d})$ is $\mathbf{r}\left(\nu_{2}\right)$-homogeneous of degree $\nu_{2}>0$ and globally fixed-time stable with respect to any ball $B_{r\left(\nu_{2}\right)}(\rho)$ with $\rho>0$ for $\tilde{d}=0$. Take $\tilde{\mathbf{r}}=$ $\left[\begin{array}{ll}\mathbf{r}\left(\nu_{2}\right) & \mathbf{r}\left(\nu_{2}\right)+\nu_{2}\end{array}\right]^{T}$, then all conditions of Theorem 2 are true and the system $\dot{x}=f_{\nu_{2}}(x, \tilde{d})$ is ISS with respect to $d \in \mathcal{L}_{\infty}$. Since $\dot{x}=f_{\nu_{2}}(x, \tilde{d})$ is the approximation of (2), (3) for $\|x\| \geq M$, then (2), (3) (the system $\dot{x}=f_{\nu(\|x\|)}(x, \tilde{d})$ ) is ISpS.

Thus, the presented state control (3) solves the posed problem of robust global fixed-time stabilization for the system (2).

\section{B. State observer}

To explain the idea let us consider first the case $d=v=0$, then the proposed observer takes the form (see also [12], [13]):

$$
\begin{gathered}
\dot{z}(t)=A_{0} z(t)+b u(t)+k(\nu(\zeta(t)), y(t)-C z(t)), \\
k_{i}(\nu, e)=L_{i}\lceil e\rfloor^{\beta_{i}(\nu)}, \beta_{i}(\nu)=1+i \nu \quad i=\overline{1, n}, \\
\dot{\zeta}(t)=-0.5 \zeta(t)+p(\nu(\zeta(t)), y(t)-C z(t)), \\
p(\nu, e)=4 \kappa^{T}(\nu, e) P \kappa(\nu, e), \kappa(\nu, e)=L e-k(\nu, e),
\end{gathered}
$$

where $z(t) \in \mathbb{R}^{n}$ is the state estimate, $\zeta(t) \in \mathbb{R}_{+}$is an auxiliary time function; the function $\nu$ is given in (4) with $-\infty<\nu_{1}<0<\nu_{2}<+\infty$ and $0<m<M<+\infty$, are, as previously, the tuning parameters; $L=\left[L_{1}, \ldots, L_{n}\right]^{T}$ is the vector of coefficients of the observer providing Hurwitz property of the matrix $A_{0}-L C ; P \in \mathbb{R}^{n \times n}$ is a matrix solution of the equations

$$
P=P^{T}>0,\left(A_{0}-L C\right)^{T} P+P\left(A_{0}-L C\right)=-P .
$$

In [13] instead of using an auxiliary $\zeta$-filter to commute the right-hand sides of (5) with negative and positive homogeneity degree, a time switching between two systems with positive and negative homogeneity degree is proposed.

Lemma 3. Let $A_{0}-L C$ be a Hurwitz matrix for a given $L \in \mathbb{R}^{n \times 1}$ and assume that the solutions of (2) are defined for all $t \geq 0$, then for any $0<m<M<+\infty$ there exists $\epsilon \in\left(0, n^{-1}\right)$ such that if $\nu_{1} \in(-\epsilon, 0)$ and $\nu_{2} \in(0, \epsilon)$ then the system (2), (5) for $d=v=0$ is globally fixed-time stable with respect to the set $\mathcal{A}=\left\{(x, z, \zeta) \in \mathbb{R}^{2 n+1}: x=z\right\}$ provided that $\zeta(0)>M$ is sufficiently big.
A more precise restriction on the value of $\zeta(0)$ is given in the proof's sketch of this lemma, it is not related with the initial conditions $x(0), z(0)$, see (7).

Proof's sketch. I Denote $e=x-z$ as the estimation error, then the error dynamics can be represented as

$$
\dot{e}=\left(A_{0}-L C\right) e+\kappa(\nu(\zeta), C e+v)+d-L v .
$$

Consider a Lyapunov function $V(e)=e^{T} P e$, then

$$
\begin{aligned}
\dot{V} & =-V+2 e^{T} P[\kappa(\nu(\zeta), C e+v)+d-L v] \\
& \leq-0.5 V+p(\nu(\zeta), C e+v)+4(d-L v)^{T} P(d-L v) .
\end{aligned}
$$

Similarly to the proof of Theorem 2 for $d=v=0$ and for any selection of $0<m<M<+\infty$ there exist $\nu_{1}, \nu_{2}$ sufficiently small such that for any $\nu \in\left[\nu_{1}, \nu_{2}\right], p(\nu, e)$ is sufficiently close to zero and

$$
\dot{e}=A_{0} e-k(\nu, C e)
$$

is globally asymptotically stable. In addition

$$
\dot{V}(e)<0 \forall e \in\left\{e \in \mathbb{R}^{n}: m \leq V(t) \leq M\right\},
$$

for any selection of any (possibly time-varying) value of $\nu(\zeta(t)) \in\left[\nu_{1}, \nu_{2}\right]$.

II For $\nu=\nu_{1}$ (6) is $\mathbf{r}\left(\nu_{1}\right)$-homogeneous of degree $\nu_{1}<0$ and globally finite-time stable at the origin, and for $\nu=\nu_{2}$ the system (6) is $\mathbf{r}\left(\nu_{2}\right)$-homogeneous of degree $\nu_{2}>0$ and globally fixed-time stable with respect to any ball $B_{r\left(\nu_{2}\right)}(\rho)$ with $\rho>0$.

III For an auxiliary error variable $\iota=V-\zeta$ we obtain:

$$
i \leq-0.5 \iota+4(d-L v)^{T} P(d-L v)
$$

and $\iota$ is exponentially converging to zero $(\zeta$ is converging to $V)$ if $d=v=0$.

Assume that $\zeta(0)>V(0)$ and denote by $T_{M}>0$ the uniform settling-time of convergence to the ball $B_{r\left(\nu_{2}\right)}\left(\rho_{M}\right)$ of (6) for $\nu=\nu_{2}$, where $\rho_{M}>0$ is such that $\left\{e \in \mathbb{R}^{n}: V(e) \leq M\right\} \subset$ $B_{r\left(\nu_{2}\right)}\left(\rho_{M}\right)$. Let

$$
T_{M}<2 \ln \left(\frac{\zeta(0)}{M}\right),
$$

then $\zeta(t) \geq M$ for $t \in\left[0, t_{M}\right)$ with $t_{M} \geq T_{M}$ ( $t_{M}$ can also be infinite). By the properties of dynamics of $\iota$ an $\zeta$, the instant $t_{M}<+\infty$ and there is another time instant $t_{m} \geq \max \left\{t^{\prime}, T_{m}\right\}$ such that $\zeta(t) \leq m$ for all $t \geq t_{m}$ $(\zeta(t)$ is exponentially approaching $V(e(t))$ from above, while $V(e(t))<m$ for $\left.t \geq T_{m}\right)$. Consequently, $\nu(\zeta(t))=\nu_{1}$ for $t \geq t_{m}$ and it reaches for the origin in a uniform time.

Corollary 2. Let all conditions of Lemma 3 be satisfied, then the system (2), (5) is ISpS with respect to the set $\mathcal{A}$ for any $(d, v) \in \mathcal{L}_{\infty}$.

Proof. Skipped due to space restrictions. 

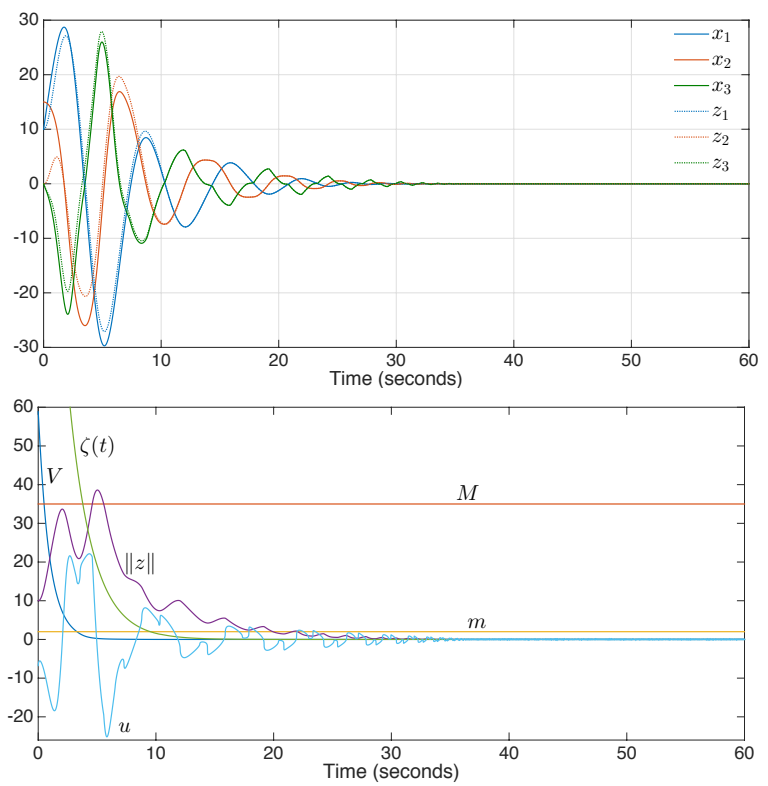

Figure 1: Results of simulation of (2), (5) and (8) for $n=3$ without perturbations and with initial conditions $x_{0}=(10,15,0)$.

\section{Output feedback}

The proposed dynamic output feedback consists in the application of the state feedback (3) with the state estimates generated by the observer (5):

$$
u(z)=\sum_{i=1}^{n} a_{i}\left\lceil z_{i}\right\rfloor^{\alpha_{i}(\nu(\|z\|))},
$$

then the dynamics of the closed-loop system (2), (5), (8) can be written in the coordinates $x, e=x-z$ and $\zeta$ as follows:

$$
\begin{aligned}
\dot{x} & =A_{0} x+b \sum_{i=1}^{n} a_{i}\left\lceil x_{i}-e_{i}\right\rfloor^{\alpha_{i}(\nu(\|x-e\|))}+d, \\
\dot{e} & =A_{0} e-k(\nu(\zeta), C e+v)+d, \\
\dot{\zeta} & =-0.5 \zeta+p(\nu(\zeta), C e+v) .
\end{aligned}
$$

The main result is a direct consequence of lemmas 2, 3 and corollaries $1,2$.

Theorem 3. Let the following conditions be satisfied:

(i) $a \in \mathbb{R}^{n}$ forms a Hurwitz polynomial;

(ii) $A_{0}-L C$ is a Hurwitz matrix for given $L \in \mathbb{R}^{n}$;

(iii) $\zeta(0)>M$ is sufficiently big,

then for any $0<m<M<+\infty$ there exists $\epsilon \in\left(0, n^{-1}\right)$ such that for $\nu_{1} \in(-\epsilon, 0)$ and $\nu_{2} \in(0, \epsilon)$ the system (2), (5), (8) is

1) fixed-time stable with respect to the set $\{(x, z, \zeta) \in$ $\left.\mathbb{R}^{2 n+1}: x=z=0\right\}$ for $d=v=0$ and for any initial conditions $(x(0), z(0)) \in \mathbb{R}^{2 n}$,

2) ISpS for any $(d, v) \in \mathcal{L}_{\infty}$.

Proof's sketch. The system (9) is a cascade of the $(e, \zeta)$ and $x$-dynamics. If $d=v=0$ then $(e, \zeta)$-subsystem is autonomous and globally fixed-time stable with respect to the set $\left\{(e, \zeta) \in \mathbb{R}^{n+1}: e=0\right\}$ (with the uniform settling-time
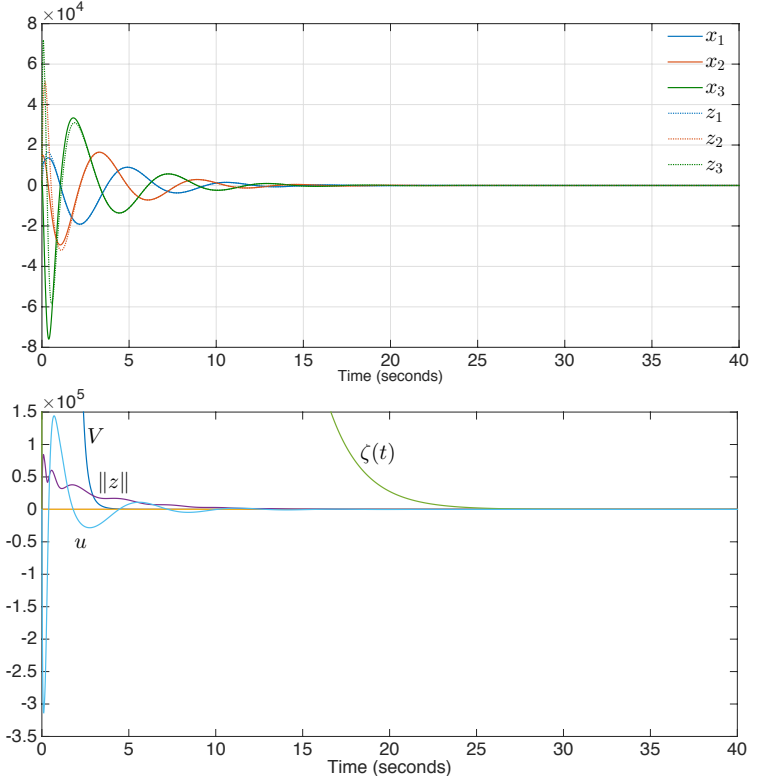

Figure 2: Results of simulation of (2), (5) and (8) for $n=3$ with initial conditions $x_{0}=10^{3}(15,10,2)$.
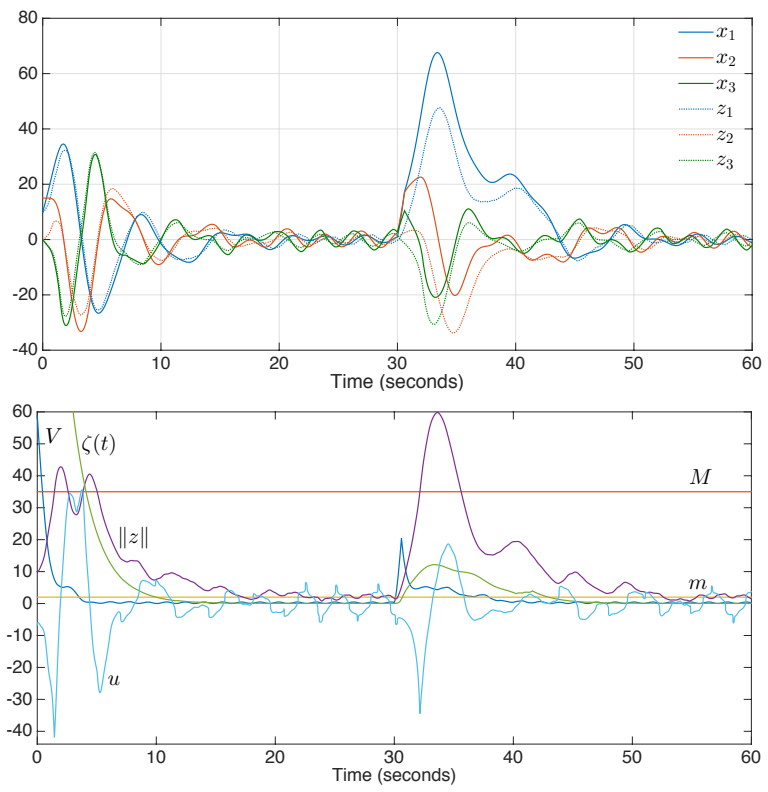

Figure 3: Results of simulation of (2), (5) and (8) for $n=3$ with the perturbation $d(t)$.

$\left.T_{o}>0\right)$ according to Lemma 3 . During the interval $\left[0, T_{o}\right]$ the system (2) has bounded trajectories due to the ISpS property with respect to measurement noises (estimation errors $e$ ) established in Corollary 1 , and for $t \geq T_{o}$ the $x$-subsystem is also autonomous and globally fixed-time converging at the origin by Lemma 2 .

For $(d, v) \in \mathcal{L}_{\infty}$ the ISpS property follows the results of corollaries 1, 2 and the cascade structure of (9).

In Theorem 3, the same parameters $m, M, \nu_{1}$ and $\nu_{2}$ have been selected for the controller (8) and for the observer (5) in order to keep the notation compact, they can be chosen 
differently in applications and the result of Theorem 3 stays correct.

\section{NumERICAL EXAMPLE}

Let $n=3, L=\left[\begin{array}{lll}1.5 & 1.01 & 0.25\end{array}\right]^{T}, a=-2.5[111]$, $v(t)=\sin (10 t), m=2, M=35, \nu_{2}=-\nu_{1}=\frac{1}{4}, \zeta(0)=$ $5 M$ and

$$
P=\left[\begin{array}{ccc}
0.121 & -0.13 & 0.047 \\
-0.13 & 0.261 & -0.308 \\
0.047 & -0.308 & 0.617
\end{array}\right],
$$

then all conditions of Theorem 3 are satisfied. We test first the system without any perturbations; the results are depicted in the upper plot of Figure 1 where the initial conditions of the system are $x_{0}=(10,15,0)$ and those of the observer are $z_{0}=(10,0,0)$. The solid color lines represent the actual state $x$ while the doted color lines represent the estimated state $z$. It can be seen how the estimated states converge rapidly to the actual states before converging both to zero. In the lower part of Figure 1 we can appreciate the elements of the control scheme, the upper and lower limits of the homogeneity degree $M$ and $n$ are shown as straight lines. The norm of the observed states $\|z\|$ is depicted in purple, while this norm is between $M$ and $m$ the control's degree of homogeneity lies over the line $\frac{\nu_{2}-\nu_{1}}{M-m}\|z\|+\frac{M \nu_{1}-m \nu_{2}}{M-m}$. In the case of the observer, the filter $\zeta(t)$ acts as the modulator of the observer's homogeneity degree. The control signal is shown in light blue.

Figure 2 shows the same setup with initial conditions $x_{0}=10^{3}(10,15,2)$, it can be seen that although the initial state is significantly larger, the settling time remains within the same interval, showing the expected uniformity w.r.t the initial state. In the lower part of the figure it can be seen that the control signal $u$ grows considerably to cope with the conditions imposed.

We next go back to the previous initial settings and introduce in the control scheme the disturbance

$$
d(t)=3 \sin (2 t)+ \begin{cases}20 & \text { if } t \in[16,17] \\ 0 & \text { otherwise }\end{cases}
$$

The results are shown in Figure 3. We can notice that the system is robust against this disturbance and its effect in the control scheme elements are depicted in the lower plot of this figure. In particular we can see that the disturbance modifies both $\|z\|$ and $\zeta(t)$ therefore changing the homogeneity degree of both the controller and the observer.

The Implicit Lyapunov function approach [9] can be used for parameter tuning of the proposed control and estimation algorithms.

\section{CONCLUSION}

A state feedback control has been constructed for a chain of integrators which ensures global convergence of all trajectories to the origin with an upper bound of the settling-time, independently of the initial conditions (fixed-time stability). An observer has been proposed, which provides a global estimation of the plant state (global differentiation) with a fixed-time convergence rate. Both control and estimation algorithms are robust with respect to disturbances and noises. It has been shown that the combination of these algorithms results in a global fixed-time output stabilization control law. The efficacy of this scheme has been demonstrated in simulations.

\section{REFERENCES}

[1] J. Doyle, B. Francis, and A. Tannenbaum, Feedback Control Systems. Mac Millan Publishing Co, 1992.

[2] A. van der Schaft, $L_{2}$-gain and passivity techniques in nonlinear control, vol. 218 of Lecture Notes in Control and Information Sciences. London: Springer-Verlag London Ltd., 1996.

[3] A. Bacciotti and L. Rosier, Lyapunov Functions and Stability in Control Theory. Springer, 2nd ed., 2005.

[4] E. D. Sontag, "The ISS philosophy as a unifying framework for stability-like behavior," in Nonlinear control in the year 2000, Vol. 2 (Paris), vol. 259 of Lecture Notes in Control and Inform. Sci., pp. 443467, London: Springer, 2001.

[5] S. Dashkovskiy, D. Efimov, and E. Sontag, "Input to state stability and allied system properties," Automation and Remote Control, vol. 72, no. 8, pp. 1579-1614, 2011.

[6] E. Moulay and W. Perruquetti, "Finite time stability of non linear systems," in IEEE Conference on Decision and Control, (Hawaii, USA), pp. 3641-3646, 2003.

[7] E. Roxin, "On finite stability in control systems," Rendiconti del Circolo Matematico di Palermo, vol. 15, pp. 273-283, 1966.

[8] A. Polyakov, "Nonlinear feedback design for fixed-time stabilization of linear control systems," IEEE Transactions on Automatic Control, vol. 57, no. 8, pp. 2106-2110, 2012.

[9] A. Polyakov, D. Efimov, and W. Perruquetti, "Robust stabilization of mimo systems in finite/fixed time," International Journal of Robust and Nonlinear Control, vol. 26, no. 1, pp. 69-90, 2016.

[10] H. K. Khalil, Nonlinear Systems. NJ 07458, Upper Saddle River: Prentice-Hall, 1996.

[11] B. M. Chen, Z. Lin, and Y. Shamash, Linear systems theory: $a$ structural decomposition approach. Springer Science \&amp; Business Media, 2004.

[12] E. Cruz-Zavala, J. Moreno, and L. Fridman, "Uniform robust exact differentiator," IEEE Transactions on Automatic Control, vol. 56, no. 11, pp. 2727-2733, 2011.

[13] M. T. Angulo, J. A. Moreno, and L. Fridman, "Robust exact uniformly convergent arbitrary order differentiator," Automatica, vol. 49, no. 8, pp. 2489-2495, 2013.

[14] A. Polyakov, D. Efimov, and W. Perruquetti, "Finite-time and fixedtime stabilization: Implicit lyapunov function approach," Automatica, vol. 51, pp. 332-340, 2015.

[15] M. Basin, Y. Shtessel, and F. Aldukali, "Continuous finite- and fixedtime regulators," in 2016 14th International Workshop on Variable Structure Systems (VSS), pp. 120-125, June 2016.

[16] E. Bernuau, D. Efimov, W. Perruquetti, and A. Polyakov, "On homogeneity and its application in sliding mode," International Journal of Franklin Institute, vol. 351, no. 4, pp. 1866-1901, 2014.

[17] V. Zubov, "On systems of ordinary differential equations with generalized homogenous right-hand sides," Izvestia vuzov. Mathematica., vol. 1, pp. 80-88, 1958 (in Russian).

[18] V. Andrieu, L. Praly, and A. Astolfi, "Homogeneous Approximation, Recursive Observer Design, and Output Feedback," SIAM J. Control Optimization, vol. 47, no. 4, pp. 1814-1850, 2008.

[19] D. Efimov and W. Perruquetti, "Conditions of oscillations and multihomogeneity," Mathematics of Control, Signals, and Systems, vol. 28, no. 3, pp. 1-37, 2016.

[20] E. Bernuau, A. Polyakov, D. Efimov, and W. Perruquetti, "Verification of iss, iiss and ioss properties applying weighted homogeneity," Systems \& Control Letters, vol. 62, no. 12, pp. 1159-1167, 2013. 